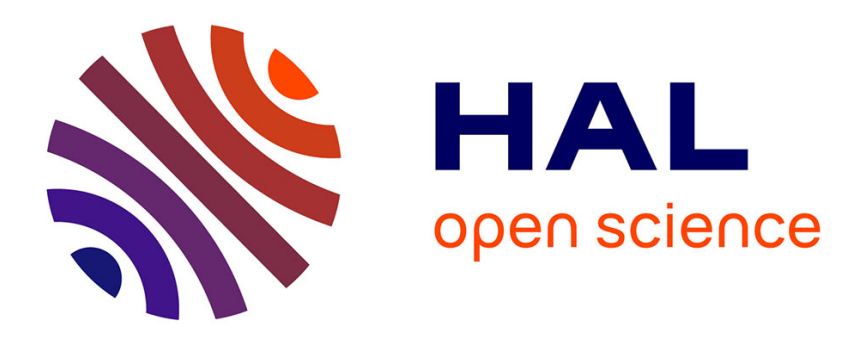

\title{
L'avenir obscur du Protocole de Kyoto
}

Moise Tsayem Demaze

\section{To cite this version:}

Moise Tsayem Demaze. L'avenir obscur du Protocole de Kyoto. 2012, pp.369-373. halshs-00773173

\section{HAL Id: halshs-00773173 \\ https://shs.hal.science/halshs-00773173}

Submitted on 11 Jan 2013

HAL is a multi-disciplinary open access archive for the deposit and dissemination of scientific research documents, whether they are published or not. The documents may come from teaching and research institutions in France or abroad, or from public or private research centers.
L'archive ouverte pluridisciplinaire HAL, est destinée au dépôt et à la diffusion de documents scientifiques de niveau recherche, publiés ou non, émanant des établissements d'enseignement et de recherche français ou étrangers, des laboratoires publics ou privés. 


\title{
L’avenir obscur du Protocole de Kyoto
}

\author{
Moïse Tsayem Demaze \\ Moise.Tsayem_Demaze@univ-lemans.fr \\ Maître de Conférences en géographie \\ UNAM, Université du Maine \\ UMR CNRS 6590 ESO, équipe ESO Le Mans
}

\begin{abstract}
Résumé
L'avenir du Protocole de Kyoto demeure ambigu alors que des négociations sont menées depuis 2005 pour la période post 2012. Aux deux groupes de travail qui menaient ces négociations, s’est ajouté un troisième groupe, créé lors de la Conférence de Durban en décembre 2011. Cette Conférence a entériné le principe d'une deuxième période d'application du Protocole. Elle a lancé des négociations pour un nouveau traité international censé entrer en application en 2020
\end{abstract}

\section{Mots-clés}

Protocole de Kyoto, conférence des parties, meeting des parties, COP, MOP

\begin{abstract}
The future of the Kyoto Protocol remains ambiguous while negotiations have been carried out since 2005. During the Durban Conference in December 2011, a third group of negotiations have been created. It is added to the two working groups that have led the negotiations so far. The Durban Conference endorsed the principle of a second period of application of the Protocol. It launched negotiations for a new international treaty intended to come into force in 2020
\end{abstract}

\section{Keys-words}

Kyoto Protocol, conference of parties, meeting of parties, COP, MOP

\section{Introduction}

La Convention Cadre des Nations unies sur les changements climatiques a été adoptée en 1992, avec pour objectif d'amener les États à «stabiliser [...] les concentrations de GES dans l'atmosphère à un niveau qui empêche toute perturbation anthropique dangereuse du système climatique ». Cette Convention a demandé aux pays développés (pays dits de l'Annexe 1) de prendre des engagements de réduction de leurs EGES, pour les ramener à leur niveau de 1990. Elle n’a ni précisé la quantité des réductions à effectuer par les pays concernés, ni indiqué la période durant laquelle ces réductions devaient être effectuées. Pour le suivi de sa mise en œuvre, elle a créé la Conférence des Parties (COP), qui est une instance rassemblant chaque année, généralement entre novembre et décembre, les États qui ont ratifié la Convention.

Au cours de la COP de 1997 à Kyoto, le Protocole de Kyoto a été adopté. Il chiffre les réductions des EGES par pays concerné et précise qu'elles doivent être effectuées entre 2008 et 2012, l'objectif global étant de parvenir à une réduction d'au moins 5\% par rapport aux émissions de l'année 1990. Le Protocole indique que la période 2008-2012 est la première période d'application des engagements de réduction des EGES (période Kyoto 1 ou PK 1). Il ne définit aucun engagement de réduction au-delà de 2012. Mais il évoque des périodes ultérieures à 2012, et prévoit la définition et l'adoption d'engagements de réduction des EGES pour ces périodes post 2012, en indiquant que l'examen de ces engagements doit commencer 7 ans au moins avant la fin de l'année 2012 (article 3, alinéa 9). Le Protocole prévoit même l’éventualité qu’un pays qui a réduit ses EGES au-delà de ses engagements pour une période, puisse faire valoir la différence durant les périodes 
ultérieures (alinéa 13 de l'article 3). Ainsi, si un pays réduit ses EGES de 10000 tonnes entre 2008 et 2012 alors qu'il avait pris l'engagement de les réduire de 8000 tonnes durant cette période, il pourra disposer d'une quantité d'émissions de 2000 tonnes à faire valoir durant la période d’engagement ultérieure à 2012 (période Kyoto 2 ou PK 2). Comme pour la Convention, un cadre a été créé pour le suivi de la mise en œuvre du Protocole. C'est le Meeting des Parties (MOP). Il rassemble les pays qui ont ratifié le Protocole. C’est une réunion qui a lieu tous les ans, conjointement avec la COP.

Le Protocole a complété et renforcé la Convention. Entré en vigueur en février 2005, il est devenu le principal instrument qui organise la lutte internationale contre le changement climatique, bien qu'il soit critiqué et jugé inefficace ou insuffisant, d'autant plus qu'il n’a pas été ratifié par les États-unis (20\% des émissions mondiales de $\mathrm{CO}_{2}$ en 2007), et qu'il ne prescrit pas de réduction aux pays émergents comme la Chine (22\% des émissions mondiales de $\mathrm{CO}_{2}$ en 2007). A ces critiques s'ajoute l'incertitude sur l'avenir du Protocole. Alors que la période Kyoto 1 arrive à terme à la fin de 2012, les contours et les engagements de réduction des EGES pour la période post 2012 ne sont pas clairement définis. Comment en est-on arrivé là ? Que révèle cette situation internationale caractérisée par l'absence de perspectives claires ? La lecture des comptes rendus et des rapports sur les négociations internationales sur la lutte contre le changement climatique, et le suivi de l'évolution récente des débats menés dans le cadre des COP et des MOP, ont permis de rédiger cette note d'humeur dont l'objectif est de montrer et d'expliquer l'ambiguïté qui caractérise l'avenir du Protocole de Kyoto.

\section{Du premier au deuxième groupe de travail spécial pour les engagements post 2012}

La question de l'application du Protocole au-delà de 2012 a été posée dès le premier MOP qui a eu lieu en décembre 2005 à Montréal conjointement avec la $11^{\text {ème }}$ COP. Lors de ce premier MOP, un groupe de travail, dénommé «Ad Hoc Working Group on Further Commitments for Annex I Parties under the Kyoto Protocol (AWG-KP), traduit en français par Groupe de Travail Spécial des nouveaux engagements des Parties à l'Annexe 1 au titre du Protocole de Kyoto (GTS-PK), a été créé. Il a engagé les discussions et les négociations pour la définition de nouveaux engagements pour les pays développés pour la période ultérieure à 2012 (PK 2). Le GTS-PK ne concerne que les pays qui ont ratifié le Protocole. Il ne peut donc engager ni les États-unis, ni les pays émergents et les pays en développement. La portée et l'ambition de son travail ne peuvent donc être que faibles par rapport à l'objectif global de réduction mondiale des EGES. Il était censé mener et finaliser ses travaux pour qu'il y ait une PK 2 et qu'il n’y ait pas de hiatus entre la PK1 et la PK2.

Lors du $2^{\text {ème }}$ MOP, qui a eu lieu en 2006 à Nairobi, conjointement avec la $12^{\text {ème }}$ COP, les réunions du GTS-PK n'ont pas abouti à la définition d'engagements pour une éventuelle PK 2. Alors que les négociations se poursuivaient sous la houlette du GTS-PK, le Plan d'Action de Bali, adopté en 2007 à l'issue de la $13^{\text {ème }} \mathrm{COP}$, a ouvert un autre cadre de discussions et de négociations en créant un autre groupe de travail dénommé Ad Hoc Working Group on Long-term Cooperative Action under the Convention (AWG-LCA), traduit en français par Groupe de Travail Spécial sur l'Action Concertée à Long terme au titre de la Convention (GTS-ACL). Le Plan d'Action de Bali indique que le mandat du GTS-ACL est de lancer un «vaste processus pour permettre l'application intégrale, effective et continue de la Convention par une action concertée à long terme, dès à présent, d'ici à 2012 et au-delà, en vue de parvenir d'un commun accord à un résultat et d'adopter une décision à sa quinzième session ». Il était attendu que ce groupe de travail engage des négociations qui aboutissent à un accord pour l'application du Protocole de Kyoto au-delà de 2012, cet accord devant être adopté lors de la $15^{\text {ème }}$ COP en 2009 à Copenhague. Les États se sont entendus non pas sur un Accord, mais sur la nécessité de parvenir d'un commun accord à un Accord. 
Le GTS-ACL a été créé sans que le GTS-PK soit supprimé. Ainsi, depuis 2007, deux groupes de travail mènent parallèlement des négociations pour l'avenir du Protocole de Kyoto après 2012. Les négociations menées par le GTS-PK ambitionnent de définir de nouveaux engagements de réduction des EGES pour les pays qui ont déjà des engagements pour la période 1 du Protocole de Kyoto, alors que les négociations menées par le GTS-ACL visent à définir de nouveaux engagements pour tous les pays. Le GTS-PK est sous la houlette du Protocole de Kyoto, alors que le GTS-ACL est sous la houlette de la Convention, et implique donc tous les pays qui ont ratifié la Convention, y compris les États-unis et les pays émergents.

\section{L'intermède de Copenhague et de Cancun : des Accords politiques ambigus}

L'existence de deux cadres parallèles de négociations pour l'avenir du Protocole a entravé l'efficacité et le succès du processus de négociations, avec une dispersion des efforts, l'apparition de contradictions, de redondances et de blocages (Maljean-Dubois et Wemaere, 2012). De la sorte, la Conférence de Copenhague en 2009 ( $15^{\text {ème }}$ COP), qui devait déboucher soit sur le prolongement du Protocole pour une deuxième période, soit sur un autre Protocole ou un nouvel Accord international définissant les engagements des États pour réduire les EGES après 2012, s'est soldé par un Accord politique faible et imprécis (Dahan, 2009 ; Dahan et al., 2010). Des États, qu'ils soient développés (pays de l'Annexe 1) ou en développement (pays non Annexe 1) ont pris volontairement des engagements en annexe de l'Accord de Copenhague pour réduire leurs EGES à l'horizon 2020, sans qu'un objectif global de réduction soit fixé et sans tenir compte des préconisations du $4{ }^{\text {ème }}$ rapport du GIEC publié en 2007 et soulignant la nécessité que les pays de l'Annexe 1 réduisent leurs EGES de 25 à 40\% à l'horizon 2020 par rapport à 1990, pour éviter que l'augmentation de la température ne soit supérieure à $2{ }^{\circ} \mathrm{C}$.

La Conférence de Cancun en 2010 (16 ème $^{\text {COP) }}$ n’a pas permis une avancée importante et significative (Dahan et al., 2011 ; Tsayem, 2011). Les engagements de réduction annexés à l'Accord de Copenhague ont été pris en considération et reconduits tacitement dans les Accords de Cancun. Cependant, la portée juridique de ces Accords parait très limitée. Ils ne se substituent pas au Protocole, et ne le prolongent pas non plus. Le sort de ce Protocole a continué à être ambigu et laissé en suspens. Le mandat du GTS-PK et celui du GTS-ACL ont été prolongés avec le souhait que leurs travaux débouchent sur un Accord lors de la $17^{\text {ème }}$ COP qui a eu lieu en décembre 2011 à Durban, conjointement avec le $7^{\text {ème }}$ MOP.

\section{Le troisième groupe de travail spécial}

La Conférence de Durban, qui était l'avant dernière avant la fin de la période Kyoto 1, n’a pas non plus réglé clairement le sort du Protocole. Elle a décidé de lancer un nouveau processus en vue de l'élaboration et de l'adoption d'un Accord dont la nature est ambiguë : «a protocol, another instrument or an agreed outcome with legal force under the Convention applicable to all Parties » (un protocole, un autre instrument ou un accord convenu avec force juridique applicable à tous les pays ayant ratifié la Convention). Le principe de la poursuite des réductions des EGES par les États après 2012 a donc été entériné dans la perspective d'un nouvel Accord global attendu des négociations à mener dans le cadre plus large de la Convention. La Plateforme de Durban, qui est en quelque sorte le résultat politique de la Conférence de Durban, est un nouveau forum ou un nouveau chantier pour décider et préciser les contours de l'avenir du Protocole. Un autre groupe de travail a été créé : c'est l'Ad Hoc Working Group on the Durban Platform for Enhanced Action (AWG-EA). On peut le traduire en français par Groupe de Travail Spécial de la Plate forme de Durban pour l'Action Renforcée (GTS-AR). Il lui est demandé de finaliser son travail au plus tard en 2015 afin que le nouveau protocole, le nouvel instrument ou le nouvel accord convenu ayant force juridique, entre en vigueur à partir de 2020. Ce nouveau groupe de travail a été créé sans que les deux précédents soient supprimés. Il existe donc 3 groupes de travail qui fonctionnement parallèlement pour décider de l’avenir du Protocole. 
Sans apporter des clarifications, la Conférence de Durban a décidé qu'une deuxième période d'engagements de réduction des EGES par les États devra commencer le $1^{\mathrm{er}}$ janvier 2013 pour une durée de 5 ou de 8 ans. Elle s’achèvera alors soit en 2017, soit en 2020. Puisque l'entrée en vigueur du nouveau traité à adopter en 2015 est prévue pour 2020, un hiatus est envisageable pour la période 2018-2020 si la deuxième période d'engagements s'achève en 2017 . Pour la $2{ }^{\text {ème }}$ période qui commencera en 2013, la Conférence de Durban a pris acte des engagements des États dans le cadre de l'Accord de Copenhague et a à nouveau invité les États à soumettre des engagements jusqu’à mai 2012. Il est attendu que la prochaine COP, prévue au Qatar en décembre 2012, débouche sur l'adoption d'un Accord de portée importante finalisant les engagements et précisant les contours de la deuxième période d’engagements (2013-2017 ou 2013-2010).

\section{Conclusion}

Une grande ambiguïté caractérise l'avenir du Protocole de Kyoto après 2012 (tabl. 1). Une deuxième période d'engagements a été décidée et devra commencer en 2013, et il est prévu qu'une autre période d'engagements commence en 2020. Trois groupes de travail fonctionnent parallèlement pour définir l'avenir du Protocole, mais les négociations donnent l'impression de s'enliser et de perdre en crédibilité, peinant à déboucher sur un traité international ambitieux qui suscite l'adhésion des États par rapport à l'importance de la réduction des EGES pour atténuer le changement climatique (den Elzen et al., 2011 ; Rogelj et al., 2011 ; UNEP, 2011). Le Canada, le Japon et la Russie ont annoncé qu'ils ne s'engageront pas pour la deuxième période. D'autres pays comme l'Australie, la Nouvelle Zélande, la Norvège, la Suisse, se sont montrés peu enthousiastes et ont indiqué qu'ils se prononceront en décembre 2012 lors de la $18{ }^{\text {ème }}$ COP (Dahan et al., 2012). Les États-unis sont toujours en marge du Protocole, puisqu'ils n’ont ratifié que la Convention. L'Union Européenne reste le « poids lourd » du Protocole, ayant réussi à Durban à agréger sur sa position les pays en développement (Maljean-Dubois et Wemaere, 2012). Les pays engagés dans la période 2 représentent environ 15\% des EGES dans le monde (Dahan et al., 2012). Avec un affaiblissement considérable et des modalités opérationnelles non encore précisées, le Protocole devrait amorcer sa deuxième période d'application en 2013. Mais son avenir au-delà de 2015 est loin d'être clarifié, en particulier sur le plan juridique, les négociations devant trancher entre un Protocole (forte portée juridique), un autre instrument ou un accord convenu (avec ou sans portée juridique forte). Malgré la gravité du changement climatique pourtant reconnue quasi unanimement par la communauté internationale (ensemble des États), cette dernière s'enlise et utilise l'astuce diplomatique consistant à multiplier des groupes de travail et à repousser l'élaboration et l'adoption d'un accord ambitieux pour la réduction des EGES par les États à partir de 2013. 


\begin{tabular}{|c|c|}
\hline Années & Faits marquants \\
\hline 1992 & $\begin{array}{l}\text { Adoption de la Convention climat actant le principe de la réduction des EGES par les } \\
\text { États }\end{array}$ \\
\hline 1997 & $\begin{array}{l}3^{\text {eme }} \text { COP à l'issue de laquelle le protocole de Kyoto est adopté, chiffrant les engagements } \\
\text { de réduction des EGES par les pays développés pour la période } 2008-2012\end{array}$ \\
\hline 2005 & $\begin{array}{l}11^{\text {ème }} \text { COP et } 1^{\mathrm{er}} \text { MOP après l'entrée en vigueur du protocole de Kyoto ; début des } \\
\text { négociations pour les nouveaux engagements des pays développés pour la période post } \\
2012 \text { (création d'un groupe de travail spécial pour les négociations : AWG-KP ou GTS- } \\
\text { PK) }\end{array}$ \\
\hline 2007 & $\begin{array}{l}13^{\text {éme }} \text { COP et } 3^{\text {ème }} \text { MOP ; début des négociations pour les engagements à long terme (post } \\
2012 \text { ) pour tous les pays ayant ratifié la convention (création d'un autre groupe de travail } \\
\text { spécial pour les engagements : AWG-LCA ou GTS-ACL) }\end{array}$ \\
\hline 2009 & $\begin{array}{l}15^{\text {ème }} \text { COP et } 5^{\text {ème }} \text { MOP ; accord de Copenhague avec des engagements volontaires à } \\
\text { l'horizon } 2020 \text { pour les pays développés et pour les pays en développement, mais avec un } \\
\text { grand flou sur sa nature juridique }\end{array}$ \\
\hline 2011 & $\begin{array}{l}17^{\text {ème }} \text { COP et } 7^{\text {ème }} \text { MOP ; décisions de Durban actant une deuxième période d'application } \\
\text { du Protocole }(2013-2017 / 2020) \text { et une troisième période à partir de } 2020 \text {, sous la forme } \\
\text { d'un protocole, d'un autre instrument, ou d'un accord convenu (création d'un } 3^{\text {ème }} \text { groupe } \\
\text { de travail spécial pour les négociations : AWG-EA ou GTS-AR) }\end{array}$ \\
\hline
\end{tabular}

Tableau 1. Principales étapes des négociations pour l’avenir du Protocole de Kyoto

\section{Références bibliographiques}

Dahan A., 2009. Entre Poznan et Copenhague : le régime climatique au milieu du gué. Natures Sciences Sociétés, $\mathrm{n}^{\circ}$ 17, p. 271-282.

Dahan A., Armatte M., Buffet C., Viard-Crétat A., 2012. Plateforme de Durban : quelle crédibilité accorder encore au processus des négociations climatiques ? Rapport de recherche, Centre A. Koyé, Koyré climate series, $\mathrm{n}^{\circ}$ 4, 34 p.

Dahan A., Aykut S., Buffet C., Viard-Crétat A., 2010. Les leçons politiques de Copenhague. Faut-il repenser le régime climatique ? Rapport de recherche, Centre A. Koyé, Koyré climate series, $\mathrm{n}^{\circ} 2$, $45 \mathrm{p}$.

Dahan A., Buffet C., Viard-Crétat A., 2011. L’ère post-Copenhague du régime climatique. Le compromis de Cancun : vertu du pragmatisme ou masque de l'immobilisme ? Rapport de recherche, Centre A. Koyré, Koyré climate series, n³ 3, 41 p.

Den Elzen M.G.J., Hol A.F., Roelfsema M. (2011b) The emissions gap between the Copenhagen pledges and the $2{ }^{\circ} \mathrm{C}$ climate goal: options for closing and risks that could widen the gap. Global Environment Change, doi:10.1016/j.gloenvcha.2011.01.006.

Maljean-Dubois S., Wemaere M. (sous la direction de), 2012. Les négociations internationales du post-2012. Une lecture juridique des enjeux fondamentaux. Rapport de recherche, $182 \mathrm{p}$.

Rogelj J., Hare W., Lowe J., van Vuuren D.P., Riahi K., Matthews B., Hanaoka T., Jiang K., Meinshausen M., 2011. Emission pathways consistent with a $2{ }^{\circ} \mathrm{C}$ global temperature limit. Nature Climate Change, vol. 1, p. 413-418.

Tsayem Demaze M., 2011. Géopolitique du développement durable. Les États face aux problèmes environnementaux internationaux. Presses Universitaires de Rennes, 228 p.

UNEP, 2011. Bridging the Emissions Gap. A UNEP synthesis report, 56 p. 